\title{
Pelagic or benthic? Mode of life of the remopleuridid trilobite Hypodicranotus striatulus
}

\author{
YUTA SHIINO, OSAMU KUWAZURU, YUTARO SUZUKI, SATOSHI ONO \& CHIHIRO MASUdA
}

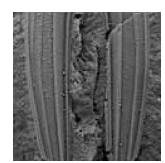

\begin{abstract}
The mode of life of the remopleuridid trilobite Hypodicranotus striatulus Walcott (1875) was examined hydrodynamically with a special focus on the relationship between the autecological performances of swimming and feeding. To understand the effect of swimming height from the sea bottom on the hydrodynamic performance of the exoskeleton, we performed computational fluid dynamics simulations on four models at differing distances from the sea bottom. The results indicated that Hypodicranotus could launch itself from the sea bottom with a relatively strong hydrodynamic lift force from slow walking or swimming speeds. However, the lift force decreased as the swimming height increased at slow swimming speeds. Hence, Hypodicranotus would have had to increase its swimming speed to greater than $0.2 \mathrm{~m} / \mathrm{s}$ and to obtain the most stable lift force at a swimming height equal to half of its own body height. Its exoskeletal morphology, with a forked hypostome, enabled it to launch itself at a slow velocity and swim at a modest distance, i.e., close to its own height, from the sea bottom. Feeding from the median vortex flows along the food groove between the two prongs of the hypostome may have been the best strategy near the sea bottom, where a large amount of food matter would have been available. Because arthropod musculature consists of striated muscles, which exhibit inferior endurance, Hypodicranotus most likely adapted to the near-bottom environment, where it could rest at times on the sea bottom as part of a nektobenthic mode of life. - Key words: biomechanics, ecomorphology, evolution, hybrid, multi-functionality, Remopleurididae, Trilobita.
\end{abstract}

ShiInO, Y., Kuwazuru, O., Suzuki, Y., ONO, S. \& Masuda, C. 2014. Pelagic or benthic? Mode of life of the remopleuridid trilobite Hypodicranotus striatulus. Bulletin of Geosciences 89(2), 207-218 (5 figures). Czech Geological Survey, Prague. ISSN 1214-1119. Manuscript received January 28, 2013; accepted in revised form November 15, 2013; published online March 11, 2014; issued May 19, 2014.

Yuta Shiino (corresponding author), The University Museum, The University of Tokyo, 7-3-1 Hongo, Bunkyo, Tokyo 113-0033, Japan; y-shiino@um.u-tokyo.ac.jp•Osamu Kuwazuru, Department of Nuclear Power and Energy Safety, University of Fukui, 3-9-1 Bunkyo, Fukui-shi, Fukui 910-8507, Japan; kuwa@u-fukui.ac.jp•Yutaro Suzuki, Institute of Geosciences, Shizuoka University, 836 Ohya, Suruga, Shizuoka, 422-8529, Japan; sysuzuk@ipc.shizuoka.ac.jp • Satoshi Ono, Department of Geosciences, Shizuoka University, 836 Ohya, Suruga, Shizuoka, 422-8529, Japan; r0934002@ipc.shizuoka.ac.jp • Chihiro Masuda, Department of Geosciences, Shizuoka University, 836 Ohya, Suruga, Shizuoka,422-8529, Japan; r0234009@ipc.shizuoka.ac.jp

Because evolution has enabled animal lineages to explore a range of adaptive strategies, understanding the autecology of fossil organisms is necessary to provide insights into their particular adaptations and how these have evolved. Swimming trilobites are appropriate candidates for this type of study because the acquisition of swimming ability from a benthic lifestyle is a significant autecological innovation (Fortey 1985, 2004). A few decades of research have demonstrated that "planktonic trilobites" commonly share certain morphological characteristics, such as a dwarfed body size and high spinosity (e.g., Fortey 1985, 2004; Clarkson \& Ahlberg 2003; Schoenemann et al. 2008, 2010), which are consistent with those of modern planktonic arthropods (e.g., Marshall \& Diebel 1995, Land 2000).
This analogy between extinct and modern taxa facilitates predictions about the mode of life of these propulsioninefficient swimming trilobites. However, the autecological characteristics of propulsion-efficient swimming trilobites remain enigmatic, particularly those of the remopleuridid trilobites. Even the generalised life mode of these animals has been classified into contrasting categories, as either benthic or pelagic (e.g., Whittington 1952, Bergström 1973, Fortey 1985, Amati et al. 2009). To resolve this question, it is necessary to provide a consistent explanation for the inter-related traits of swimming capability, habitat and the corresponding biological performance measures within the framework of the animal body plan.

The remopleuridid trilobite Hypodicranotus striatulus 

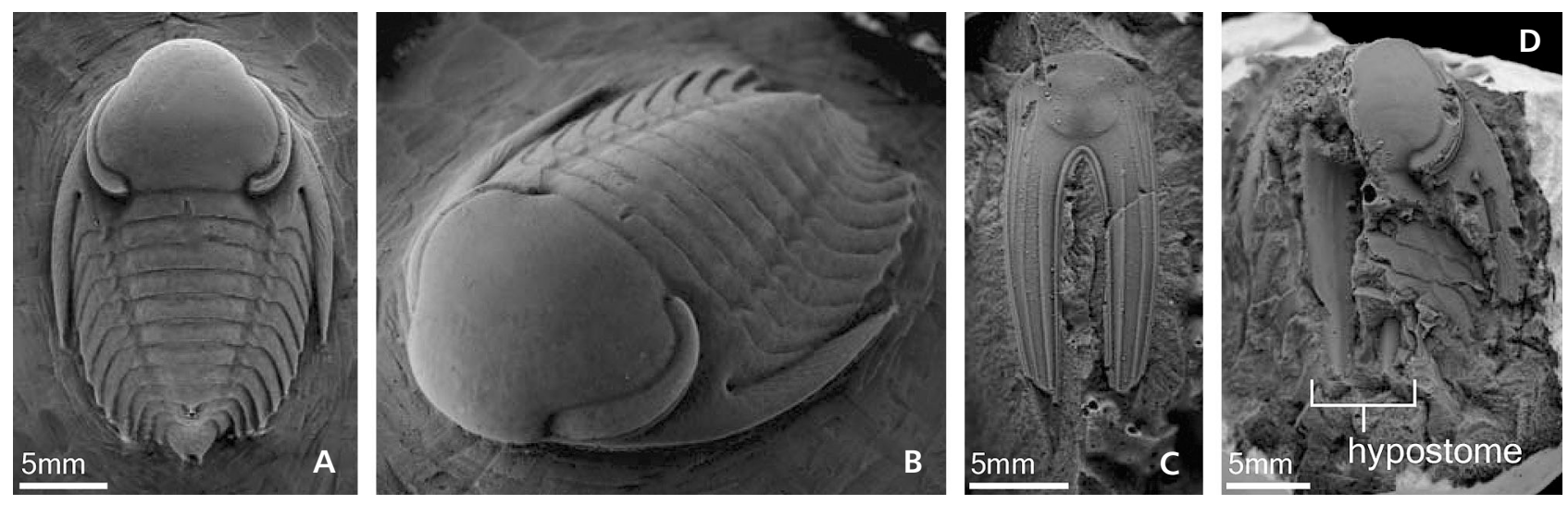

Figure 1. Morphology of Hypodicranotus striatulus. - A, B - dorsal and antero-lateral views of Hypodicranotus striatulus (MCZ-115910). - C - hypostome (MCZ-100987). $\bullet$ D - dorsal view of a left-half broken specimen (MCZ 100988) with the hypostome partially exposed on the left side.

Walcott (1875) was a propulsion-efficient swimming animal with a hydrodynamically sophisticated morphology in the Ordovician sea (Fig. 1, Shiino et al. 2012). Its exoskeletal form was highly streamlined to avoid flow detachment, and the long, bilaterally pronged hypostome reduced viscous drag while maintaining a modest lift force over a wide range of swimming speeds (Shiino et al. 2012). In addition to this hydrodynamic morphology, Hypodicranotus exhibited notable adaptations for feeding while swimming, using the flow of water through its food groove to consume tiny, suspended organic particles (Fig. 2, Shiino et al. 2012). Water flows during swimming also contributed to the respiratory ventilation of the animal (Fig. 2, Shiino et al. 2012). The occurrence of Hypodicranotus fossils in a variety of sedimentary facies and their inter-provincial palaeobiogeography has been interpreted to indicate a pelagic swimmer (Brett et al. 1999, Amati et al. 2009). In contrast, the food source utilised in this feeding style might limit adaptation to a specific environment. Therefore, it is necessary to determine how habitat differences would affect the balance of feeding, respiration and swimming behaviour that the organism needed in order to prosper.

The aim of this study was to examine the swimming performance of an exoskeletal model with respect to its swimming speed and height above the sea bottom. Computational fluid dynamics (CFD) simulations were performed to determine where the animal would have obtained the most efficient drag and lift forces. Based on the simulation results, we discuss the mode of life of Hypodicranotus with a special focus on the relationship between swimming capability and integrated aspects of biological performance.

\section{Materials and methods}

The CFD simulation methods used here have been fully described in previous reports by our group (Shiino et al.
2009, 2012; Shiino \& Kuwazuru 2010, 2011a, b). The methodology is briefly described below.

\section{Fossil specimens}

A complete, articulated dorsal exoskeleton and a hypostome of the Ordovician remopleuridid trilobite Hypodicranotus striatulus Walcott (1875) from the Trenton Group, New York, United States of America, were used for the simulation (Fig. 1A-C). These specimens are housed in the Museum of Comparative Zoology at Harvard University (MCZ-100987, MCZ-115910). The dorsal exoskeletal specimen is $25.5 \mathrm{~mm}$ long and $17.0 \mathrm{~mm}$ wide, and the hypostome is $17 \mathrm{~mm}$ long and $7.6 \mathrm{~mm}$ wide. Additionally, an exoskeletal model with a hypostome was reconstructed based on specimen MCZ 100988 (Fig. 1D).

\section{Computational fluid dynamics (CFD) simulations}

Water flows around the exoskeleton with the hypostome attached in its original position were simulated by solving the incompressible Navier-Stokes (NS) equations for unsteady viscous fluids. The simulation was performed using the commercial computational fluid dynamics code SCRYU/Tetra 9.0 (Software Cradle Co., Ltd., Tokyo, Japan), which employs the finite-volume method (e.g., Adkins \& Yan 2006, Shiino et al. 2009). To implement a three-dimensional model of Hypodicranotus for analysis in the CFD simulation, we constructed a polycarbonate exoskeletal replica using the vacuum-forming method (Shiino et al. 2009, Shiino 2010) and obtained sequential cross-sectional images of the replica using an SMX-225CT microfocus X-ray CT system (Shimadzu Corporation, Tokyo, Japan). Subsequently, the volumetric data were translated into surface morphological data in 

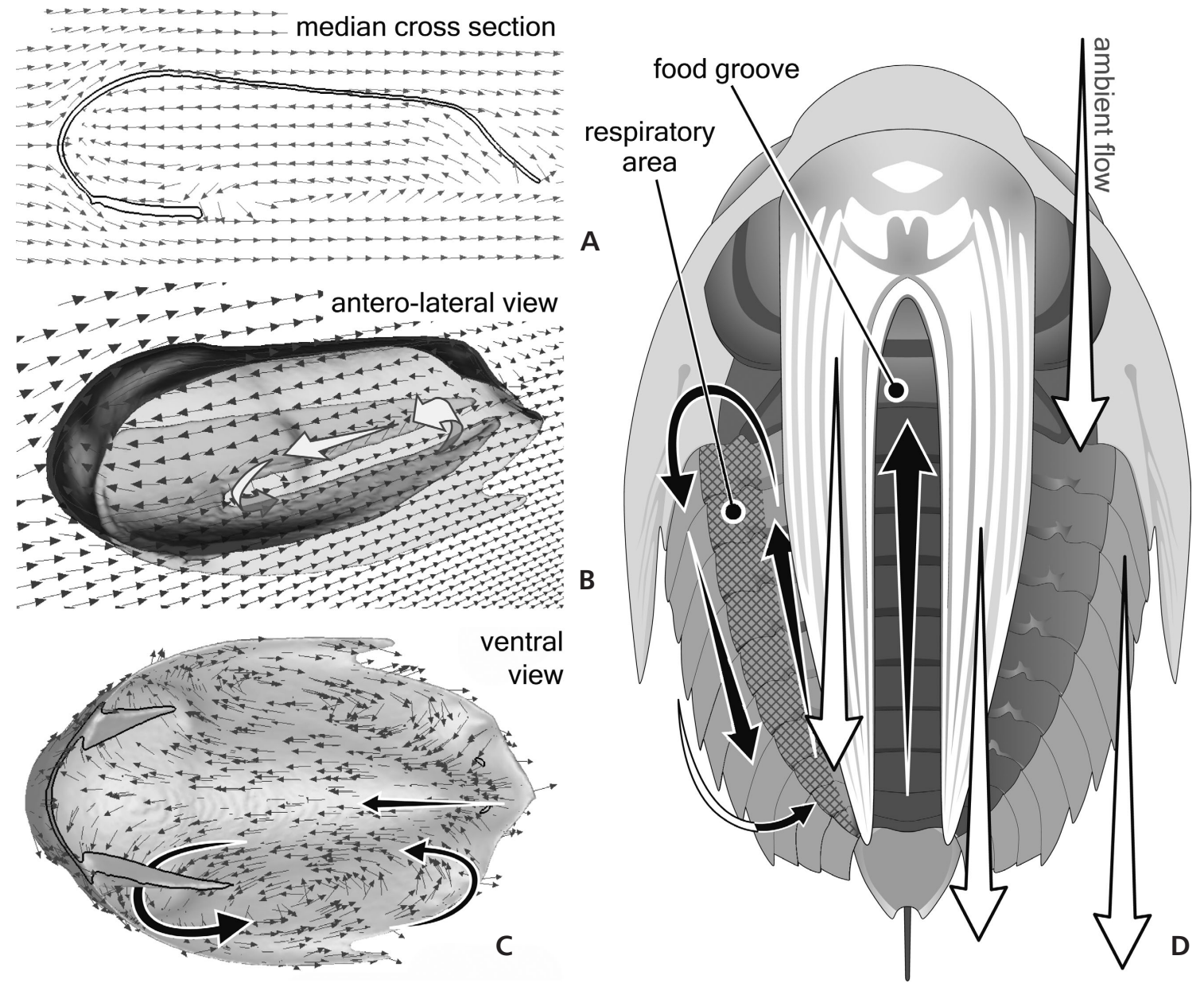

Figure 2. Flows around the hypostome-bearing model. $\bullet$ A - flow vectors in median cross sectional view. $\bullet$ B - flow vectors around the median cross section in antero-lateral view. $\bullet \mathrm{C}$ - vector representations of flows on the internal surface. The hypostome is shown as a transparency. $\bullet \mathrm{D}-$ schematic illustration of ambient and secondary flows. Swimming provides biological benefits in the form of feeding using the food groove and respiration using the counter-currents along the inferred trilobite gills (after Shiino et al. 2012).

the Standard Triangulation Language (STL file type) using the image-processing function of the image-based structural analysis software VOXELCON 2005 (Quint Corporation, Japan). The STL file was transferred into SCRYU/Tetra, and finite-volume meshes of tetrahedral elements were generated automatically (Shiino et al. 2009).

The Reynolds-averaging method can be used to decompose the pressure and velocity in the NS equations into a time average and a fluctuation component, resulting in the Reynolds-averaged Navier-Stokes (RANS) equations (e.g., Bertram 2000). Along with the RANS equations, we employed an improved $k-\varepsilon$ turbulence model based on the turbulent kinetic energy $k$ and its dissipation rate $\varepsilon$, which is known as the AKN model (Abe et al. 1992, 1995). The AKN model is applicable to consider near-wall eddy viscosity in flow fields with a low Reynolds number (Abe $e t$ al. 1992, 1995); thus, it is suitable to simulate flows around our Hypodicranotus model (Shiino et al. 2012). The total simulation time was set at 15 seconds because the fluid structure reached a stable condition with or without vortex fluctuations, similar to a Karman vortex, within this time period. We used a constant time step of 0.003 seconds to obtain a reasonable computation time. Our preliminary analyses confirmed the accuracy of simulations using these parameters (Shiino et al. 2012).

To examine the hydrodynamic properties of the exoskeleton at different distances from the sea bottom, we adopted an analysis domain consisting of a cuboid space that extended a distance of 10 times the model length in the downstream direction; 5 times the model width and height in the lateral and upward directions, respectively; and 3 times the model length in the upstream direction (Fig. 3). The distance between the model and the bottom of the space was set at $0.2,0.5$ and 1.0 times the model height $\left(L_{3} ; 8.1 \mathrm{~mm}\right)$ (Fig. 3). To evaluate the effect of flow velocity (i.e., coasting speed) on the hydrodynamic properties of the exoskeleton, we simulated 11 different uniform inlet 

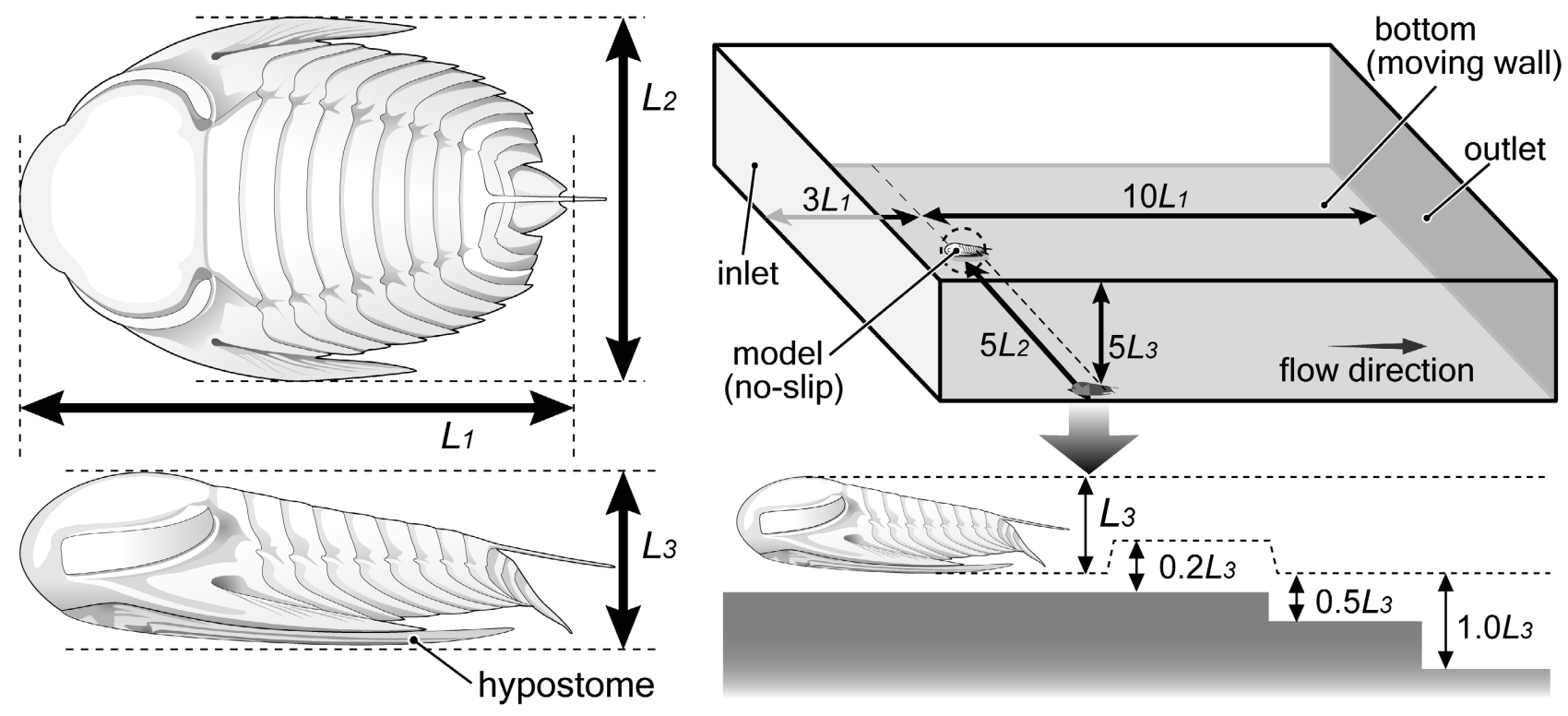

Figure 3. Schematic illustrations of the analysis domain for the computational fluid dynamics simulation showing the distances from the bottom examined in this study.

velocities, ranging from 0.01 to $0.5 \mathrm{~m} / \mathrm{s}$ (corresponding to Reynolds numbers of 270 to 13500 , where the body length of $25.5 \mathrm{~mm}$ was adopted as a representative length). The outlet pressure was fixed to zero. The boundary condition of the bottom was set as a moving wall whose direction and speed were concordant with the inlet flow (Fig. 3). All other parameters of the CFD simulation matched those used in our previous studies (Shiino et al. 2009, 2012; Shiino \& Kuwazuru 2010, 2011a, b).

\section{Evaluation of the bottom effect}

To qualitatively examine the bottom effect on the swimming capability of Hypodicranotus, we calculated the drag and lift forces as functions of the distance from the bottom. Propulsion-efficient swimming organisms, including $\mathrm{Hy}$ podicranotus, reduce their drag forces to swim easily and to obtain a modest lift force to stabilise the body horizontally (Shiino et al. 2012). To compare the hydrodynamic effects of different distances from the sea bottom at different coasting speeds, we normalised the drag and lift forces by the drag predicted from the nominal hydrodynamic pressure. We refer to the resulting normalised drag coefficient as $C_{d}$ and the lift coefficient as $C_{l}$. The coefficients were calculated as $C_{d}=2 F_{d} / \rho v_{e x}{ }^{2} S$ and $C_{l}=2 F_{l} / \rho v_{e x}{ }^{2} S$, where $F_{d}$ is the drag force, $F_{l}$ is the lift force, $\rho$ is the mass density of the fluid, $v_{e x}$ is the inlet velocity and $S$ is the projected frontal area of the model (Vogel 1994). The projected frontal area of the hypostome-bearing model was $85.47 \mathrm{~mm}^{2}$, as estimated using the SCRYU/Tetra postprocessing function.

\section{Results}

\section{Drag and lift forces and coefficients}

The drag and lift forces and the corresponding coefficients for all simulated cases are shown in Fig. 4. The drag force increased as the coasting speed increased (Fig. 4A), whereas its coefficient decreased monotonically (Fig. 4B). The drag forces and coefficients were similar regardless of the swimming height, except at the shortest distance from the bottom (Fig. 4A, B: $0.2 L_{3}$ ). The pattern of drag forces and coefficients suggests that efficient swimming could be achieved at all the heights except the lowest level $\left(0.2 L_{3}\right)$. By lifting its body away from the bottom, the animal could quickly decrease its drag and obtain suitable swimming conditions.

The lift forces and coefficients differed dramatically with respect to the distance from the bottom (Fig. 4C, D). The lift force increased as the coasting speed increased (Fig. 4C), whereas its coefficient decreased (Fig. 4D). At a coasting speed of $0.15 \mathrm{~m} / \mathrm{s}$, the lift force and coefficient increased as the body approached the bottom, suggesting a highly efficient launching ability in the initial stage of swimming near the bottom. At coasting speeds greater than $0.2 \mathrm{~m} / \mathrm{s}$, the simulated results always showed stable lift coefficients, indicating that the lift force could be readily controlled by altering the swimming speed. The lift force and coefficient were maximised near the swimming height of $0.5 L_{3}$. Thus, the bottom effect is expected to produce higher lift forces at higher velocities (Fig. 4C, D). At velocities near $0.1 \mathrm{~m} / \mathrm{s}$, the variation in the lift coefficient was smooth at the swimming height closest to the bottom 


\section{A Drag force and coefficient}
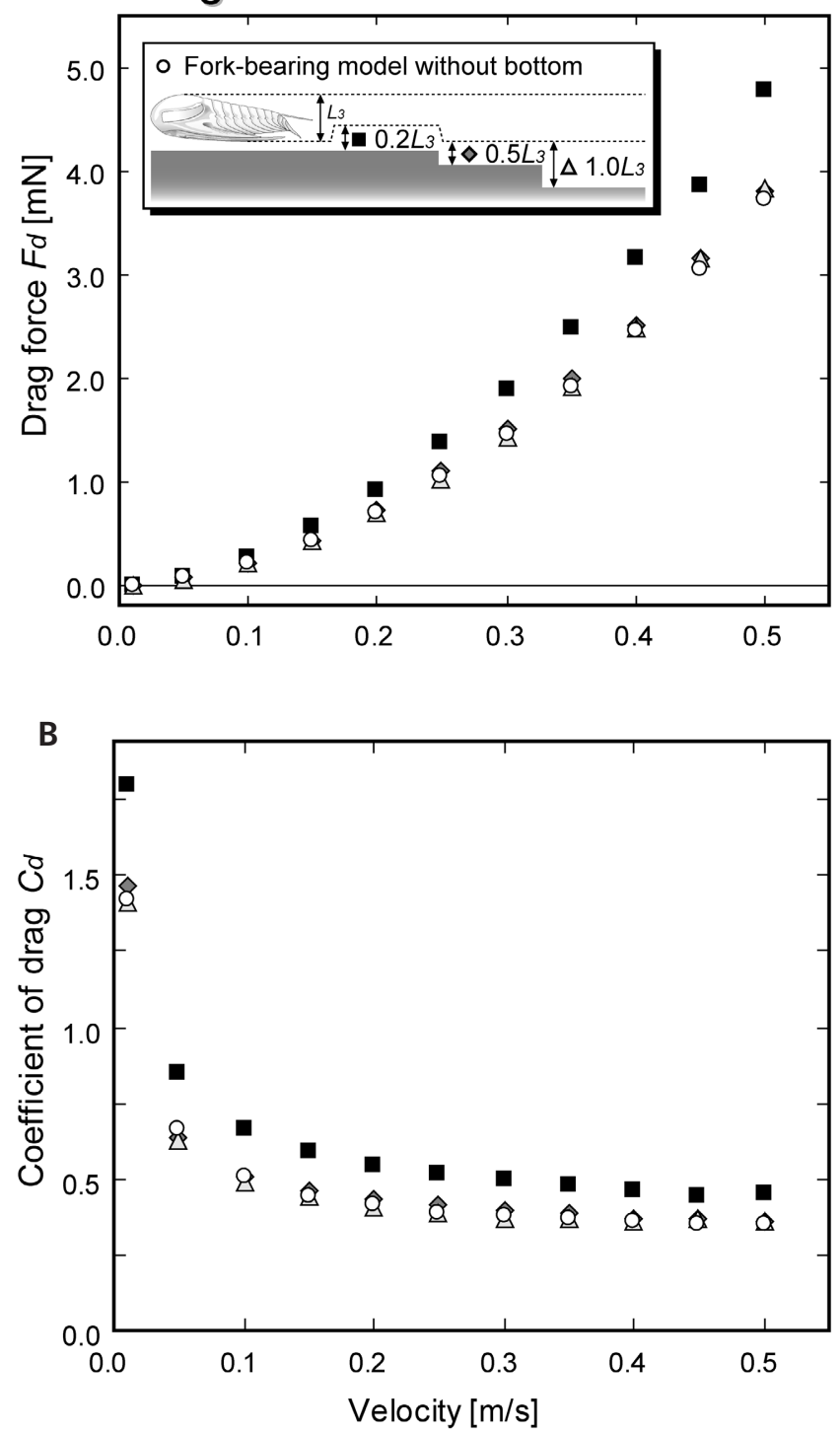
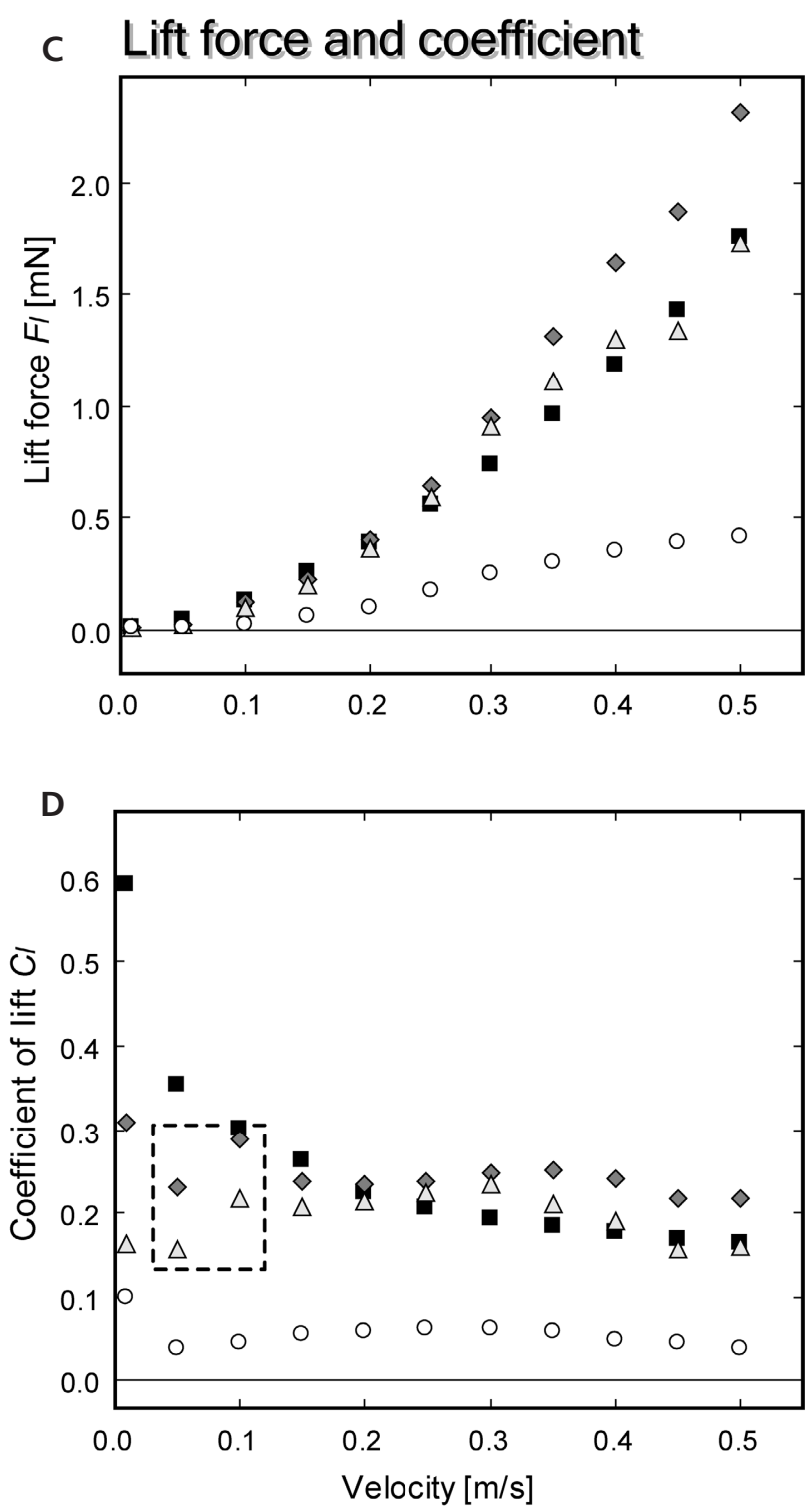

Figure 4. Drag and lift forces and coefficients at different velocities and distances from the bottom. The results for the hypostome-bearing model with no bottom effect (open circles) are from Shiino et al. (2012). $\bullet$ A - drag forces. $\bullet$ B - drag coefficients. $\bullet$ C - lift forces. $\bullet$ D - lift coefficients.

$\left(0.2 L_{3}\right)$ but not at swimming heights farther from the bottom $\left(0.5 L_{3}, 1.0 L_{3}\right)$ because the flow behind the body fluctuated due to turbulence. In other words, the flow changes from laminar to turbulent at this velocity. However, this effect strongly depends on the angle of the tail, which was modelled after the subject fossil.

The evidence presented here indicates that at the sea bottom, the drag force is relatively high, but the lift force is also high. Near the sea bottom, the drag force quickly declines to the magnitude it would exhibit without a bottom effect, and the lift force becomes stable at relatively high velocities. At lower coasting speeds (less than $0.15 \mathrm{~m} / \mathrm{s}$ ), the pressure beneath the model increased because flows tended to be stagnant, decreasing in velocities. This flow condition can provide higher lift coefficients near the sea bottom. At higher coasting speeds (more than $0.2 \mathrm{~m} / \mathrm{s}$ ), smooth flows beneath the model resulted in higher velocities, which created lower lift coefficients (Fig. 4D). A significant reduction of fluid pressure would be expected at the swimming height closest to the bottom $\left(0.2 L_{3}\right)$ due to flows through the constricted space responding to the Venturi effect and therefore causing the lift coefficient to drastically decrease as the coasting speed increases (Fig. 4D). A swimming height of $0.5 L_{3}$ provides the maximum efficiency needed to obtain lift force. At higher levels, the lift force also decreases and approaches the magnitude it would exhibit without a bottom effect (Fig. 4: open circles in each plot). 


\section{Discussion}

\section{Launching to swimming}

The results presented above clearly show that Hypodicranotus could swim most effectively, with lower drag and higher lift, at close distances from the bottom but not in the upper water column. Because Hypodicranotus can be regarded as a nektobenthic swimmer in terms of its hydrodynamic properties, it is necessary to explain the effect when they launched from the sea bottom.

It is generally accepted that moving the metameric appendages could provide a propulsive force for swimming (Bergström 1969, 1973; Whittington 1975). Because trilobite appendages exhibit little specialisation along the body, metachronal movements would result in drag-based propulsion, a so-called serial paddling, which are a typical mode of appendage movement observed in extant isopods and horseshoe crabs (Azuma 2006). Serial paddling is the ultimate transformation of accelerated level-bottom walking by marine arthropods. Therefore, it is expected that Hypodicranotus launched from the sea bottom with the aid of a relatively high lift force when walking.

According to Shiino et al. (2012), the presence of soft tissues may affect the magnitude of drag force to some degree. Taking the hydrodynamic influence of the appendages into consideration, the space between the body and the sea bottom would significantly decrease. In this condition, the lift force increases at lower coasting speeds by means of flow stagnation, while it decreases at higher coasting speeds, in comparison with the present simulation model. The sudden reduction of the lift coefficient at the swimming height closest to the sea bottom $\left(0.2 L_{3}\right)$ would be further emphasised by the Venturi effect as mentioned above. Such instability of the lift coefficient near the bottom seems to be unreasonable for coasting-swimming.

Metamerically arranged and laterally deflected trilobite appendages facilitate smooth fluid flows under the pleural fold. This grants Hypodicranotus stability while swimming and a way to lift their body above the sea bottom during resting or walking. Our results suggest Hypodicranotus could move through a stable and smooth sequence of walking, launching and swimming at a height equal to half of its own body height $\left(0.5 L_{3}\right)$.

\section{Fossil evidence and relevant sedimentary environment}

Based on its lack of lithofacies dependency, it has been suggested that Hypodicranotus was a pelagic swimmer (Amati et al. 2009). The relevant sedimentary environments of Hypodicranotus fossils suggest habitats ranging from shallow-ramp to slope settings (Amati et al. 2009), in- dicating that the animal was not particularly adapted to the sedimentary facies and bottom environments (Amati \& Westrop 2006, Amati et al. 2009). This evidence was adopted to infer a pelagic life style (Fortey 1985). Many modern organisms with swimming capability are widely distributed across provinces and habitat depths, unlike sessile benthic organisms. Nevertheless, not all modern swimming organisms are pelagic. Thus, the inference that an organism with no obvious dependence on the conditions of the sea bottom must be pelagic may mislead autecological interpretation. For example, the benthic shrimp species Solenocera melantho is distributed at depths of 70 to $400 \mathrm{~m}$ in its shelf to lower-slope habitat (Chan 1998). Although this species is restricted to the Western Pacific, the genus Solenocera exhibits a wider range of depths across its global distribution (e.g., Kunju 1970, Holthuis 1980). Similarly, the isopod species Bathynomus doederleinii inhabits depths of 100 to $686 \mathrm{~m}$ in the Western Pacific, and the genus Bathynomus ranges from 70 to $2140 \mathrm{~m}$ in depth across the Atlantic, Pacific and Indian Oceans (Schotte et al. 1995). Both of these modern arthropods are regarded as benthic animals with some degree of swimming ability. Thus, it is difficult to determine whether motile fossil organisms were pelagic or benthic based solely on their facies dependency, or lack thereof.

Characteristic wide distribution patterns, regardless of depth and bottom conditions, might be closely related to the autecological properties of an organism. For example, the extant shrimp Solenocera depends on a variety of food sources, including fish, decapods, foraminifers and molluscs (Dineshbabu \& Manissery 2009). These highly variable food sources depend on the benthic environment to which each species is adapted (Dineshbabu \& Manissery 2009), resulting in a wide range of habitats and depth distributions for the genus. The isopod Bathynomus, which employs a sit-and-wait foraging strategy, also occupies a wide range of habitats because it can remain stationary, consuming little energy, until a dead organism such as a fish or a whale falls through the water column nearby (e.g., Johansen \& Brattegard 1998, Lowry \& Dempsey 2006, Matsui et al. 2011). In general, carnivorous arthropods such as Limulus use endites for grasping and ascending food particles toward mouth openings. This is achieved mechanically by repeated adductive motion of adaxial podomeres. In Hypodicranotus, the long, forked hypostome with its rigid connection to the cephalic shield would cover the adaxial podomeres of the appendages. Thus, the use of these appendages for predation would be structurally unreasonable. Instead, Hypodicranotus may have fed efficiently by capturing suspended particles from the water flowing along its food groove, as discussed previously (Shiino et al. 2012). The water flowing along the food groove could transport comparatively small organic particles. In any facies or sedimentary environment, organic particles are abundant 
around the sediment-water interface (Boucot 1981, Nowell et al. 1981, Snelgrove 1998). Although Hypodicranotus is a benthic dweller with a strong swimming capability, not a pelagic swimmer, a weaker dependence on particular food resources would enable this animal to occupy a wide range of depths.

Further explanation is required for the inter-provincial distribution pattern of Hypodicranotus, which is related to larval dispersal ability rather than swimming capability and related biological performances. The morphologically and taxonomically related genus Remopleurides has protaspides of nonadult-like globular forms called asaphoid protaspides. Unique to this form is the planktonic protaspid growth stages, and earlier meraspid stages (Chatternton \& Speyer 1997). A number of studies have precluded a benthic life style on the basis that the larval body size was less than a millimetre, with three dimensionally extending long spines (e.g., Whittington 1959, Fortey \& Chatterton 1988, Chatterton \& Speyer 1997, Park $\&$ Choi 2011). These morphological features fulfil the general requirement for a planktonic life style because it had a large surface area in relation to its volume (e.g., Ruppert et al. 2003).

With these facts in mind, one may imagine that the morphological characteristics of larval stages have facilitated the inter-provincial distribution pattern of remopleuridids. However, the explanation is not so simple because there still remains the problem of inter-provincial distribution of Hypodicranotus. The fossil occurrences of Hypodicranotus are known from North America, the United Kingdom, the Ural Mountains of Siberia and Kolyma of eastern Russia (Ludvigsen \& Chatterton 1991). The palaeogeographic position of the Kolyma Terrain during the Ordovician appears to be the Mediterranean Province, possibly near Laurentia (Zhang \& Barnes 2007, Rasmussen \& Harper 2011). In contrast to the case of Hypodicranotus, the genus Remopleurides likely had a broad distribution (Amati et al. 2009) even for supposed propulsion-efficient swimmers. The relationship between swimming capability and distribution may not be linear and needs to be further examined to reach an appropriate understanding of the remopleuridid mode of life.

\section{Biological aspects of swimming}

With this mechanical interpretation of swimming, the biological traits of trilobites may provide further insight into their modes of life. The swimming method of serial paddling takes advantage of acceleration at comparatively slow speeds for short durations (Vogel 1994, Azuma 2006). The cessation of swimming in Hypodicranotus might have resulted in sinking towards the bottom. There- fore, the serial paddling observed in arthropods would be incompatible with a pelagic mode of life without morphological adaptation enabling the frictional retardation of sinking. To explore the pelagic habitat, propulsionefficient swimming animals may acquire lift-based swimming abilities, such as the undulating fins of fish, that enables them to swim faster and for longer durations (Vogel 1994, Azuma 2006). Alternatively, propulsion-efficient swimming abilities may be lost, as observed in immobile planktonic animals.

The body plan of Hypodicranotus provides further evidence of its swimming ability. In arthropods, the muscular system consists only of striated muscles (Dorit et al. 1991). This type of muscle provides instantaneous locomotive force but is not effective for endurance (Dorit et al. 1991). Therefore, Hypodicranotus would need to rest in the water column if it were a pelagic swimmer. This behaviour appears unlikely due to the morphological features described above. Indeed, planktonic locomotion is efficient for pelagic arthropods. Slowly moving the appendages through the water may enable some arthropods to float because their exoskeletons can withstand considerable fluid resistance (e.g., Marshall \& Diebel 1995). In addition, exposing the appendages could obtain the effective drag force that contributes to further enhancement of the floating mode of life. In the case of Hypodicranotus, the long, forked hypostome covered the venter to reduce the drag force (Shiino et al. 2012), which seems in opposition to this direction of morpho-functional strategy.

\section{Feasible autecology of Hypodicranotus striatulus}

As discussed above, swimming capability and geological evidence indicate that Hypodicranotus was a non-pelagic swimmer. Because this animal may have adapted to the benthic environment through its propulsion-efficient swimming capability, further explanations of its adaptations to the nektobenthic lifestyle are needed. The feeding strategy of Hypodicranotus (see Fig. 2) would be beneficial in the benthic environment, where a large amount of detritus is available for feeding (e.g., Shiino et al. 2011). The feeding method resembles that of tadpole shrimp, in which beating the metameric appendages to swim generates flows along the food groove, and these secondary flows raise a plume of food materials from the substrate-water interface (Fryer 1988). Thus, the morphology of Hypodicranotus was adapted to collect food particles by antero-dorsally directing vortex flows along the food groove. This process would be most efficient in nektobenthic environments.

In addition to this feeding strategy, the ability to swim provided a new defensive strategy for trilobites. Through 
enrolment, some benthic trilobites could entirely encapsulate the non-mineralised parts of their bodies, protecting them against predatory attack (e.g., Esteve et al. 2010, 2011; Feist et al. 2010). We argue that this defensive strategy provided a secondary benefit for trilobites and other arthropods with enrolment or volvation abilities. Predators that use vision to detect potential prey depend on motion-capture perception; thus, they are unlikely to perceive static, enrolled individuals as potential prey. This defensive strategy is similar to thanatosis (e.g., Nishino \& Sakai 1996). Instead of compromising its enrolment ability (Hegna 2010), the swimming ability of Hypodicranotus provided an active escape mechanism, as observed in modern arthropods (e.g., Jacklyn \& Ritz 1986). Given the advantages of swimming for feeding and respiration, other aspects of biological performance, including escape behaviour, were functionally integrated with the propulsion-efficient swimming system of Hypodicranotus within its trilobite body plan.

In summary, our simulations revealed that Hypodicranotus could obtain a large and stable lift force for effortless swimming near the sea bottom. Instead of enhancing swimming performance, the swimming movement of Hypodicranotus would generate postero-anteriorly directed vortex flows beneath the ventral side of the dorsal tergites, which would initially appear to be disadvantageous (Shiino et al. 2012). Importantly, however, the animal could divert these hydrodynamic handicaps for feeding and respiration (Suzuki \& Bergström 2008, Shiino et al. 2012), thus functionally integrating the swimming, feeding and respiratory systems as well as protection (Fig. 5). Its weak dependence on specific food resources enabled this species to occupy a broad range of habitats despite its nektobenthic mode of life.

\section{Propulsion-inefficiency or -efficiency in trilobite swimming}

Because swimming behaviour is heavily influenced by surrounding viscous fluids, morphological characteristics greatly influence swimming capability and mode of life. Swimming trilobites could be subdivided into two broad types of exoskeletal morphology. One is the morphology with proportionally large cephalon against whole exoskeletal body plus spherical eyes as observed in Carolinites, Opipeuterella, Cyclopyge, Telephina and Symphysops. This morphological type must be a propulsion-inefficient swimmer because of its great surface friction. The locomotion would be slow, and the swimming speed would be only several body lengths per second. The locomotion may immediately stop at the conclusion of beating the appendages, and thus, its seemingly planktonic mode of life. The other morphological type is likely to be a propulsion- efficient swimmer because of the streamlined exoskeleton, allowing the locomotion of coasting or grinding. The number and the size of ommatidia differ greatly from that of propulsion-inefficient swimmers. Assuming these morphological requirements, remopleuridids such as Amphitryon, Remopleurides and Hypodicranotus are certainly candidates for propulsion-efficient swimmers.

\section{Future perspectives in understanding the biophysiological performance of fossil organisms}

Understanding the morphological functions that characterise animal adaptations makes it possible to reconstruct the biological traits of fossil organisms (e.g., Shiino \& Suzuki 2007, 2011; Lerosey-Aubril et al. 2011; Shiino \& Kuwazuru 2011a). Because we cannot reliably test or confirm the lifestyles of fossil organisms, it has been suggested that knowledge about their morphological functions represents the only source of information in understanding the autecological properties of fossil organisms. However, all animals must be treated as multifunctional entities to ensure the consistent integration of the various aspects of their biological performance. As the present study demonstrates, a plausible autecological scenario must explain multiple inter-related performance traits (e.g., Hughes \& Chapman 1995, Suzuki et al. 2008, Kaji et al. 2011, Fujiwara \& Hutchinson 2012, Matsumura \& Yoshizawa 2012).

Earlier misinterpretations of morphological functions were caused by the lack of reliable quantitative analyses, which can enhance our understanding of adaptive functional capabilities (Briggs et al. 1991). Trilobite morphotypes have been inferred to represent adaptations to specific lifestyles (Fortey \& Owens 1990), and these inferences provide working hypotheses about the palaeoecological characteristics of these organisms. However, exceptions to the rule of morphological adaptation, especially in swimming trilobites (Fortey 1985, p. 418 in Hughes 2007), invalidate the one-sided approach to morphotypic properties.

In the family Remopleurididae, Remopleurides has comparatively stout genal spines that extend postero-laterally and a long thoracic mid-axial spine on the eighth thoracic segment (e.g., Nikolaisen 1988). These morphological features would retard the sinking rate, as observed in planktonic animals, and are apparently different than the adaptive features of Hypodicranotus. Although the morphological features of Remopleurides seem to suggest a planktonic mode of life, their habitat was restricted around the inner shelf, unlike a simple pelagic animal (Hansen et al. 2011). The ball-socket structure of articulation between thoracic segments in Remopleurides is visibly bloated (Nikolaisen 1988); the excessive development may have 


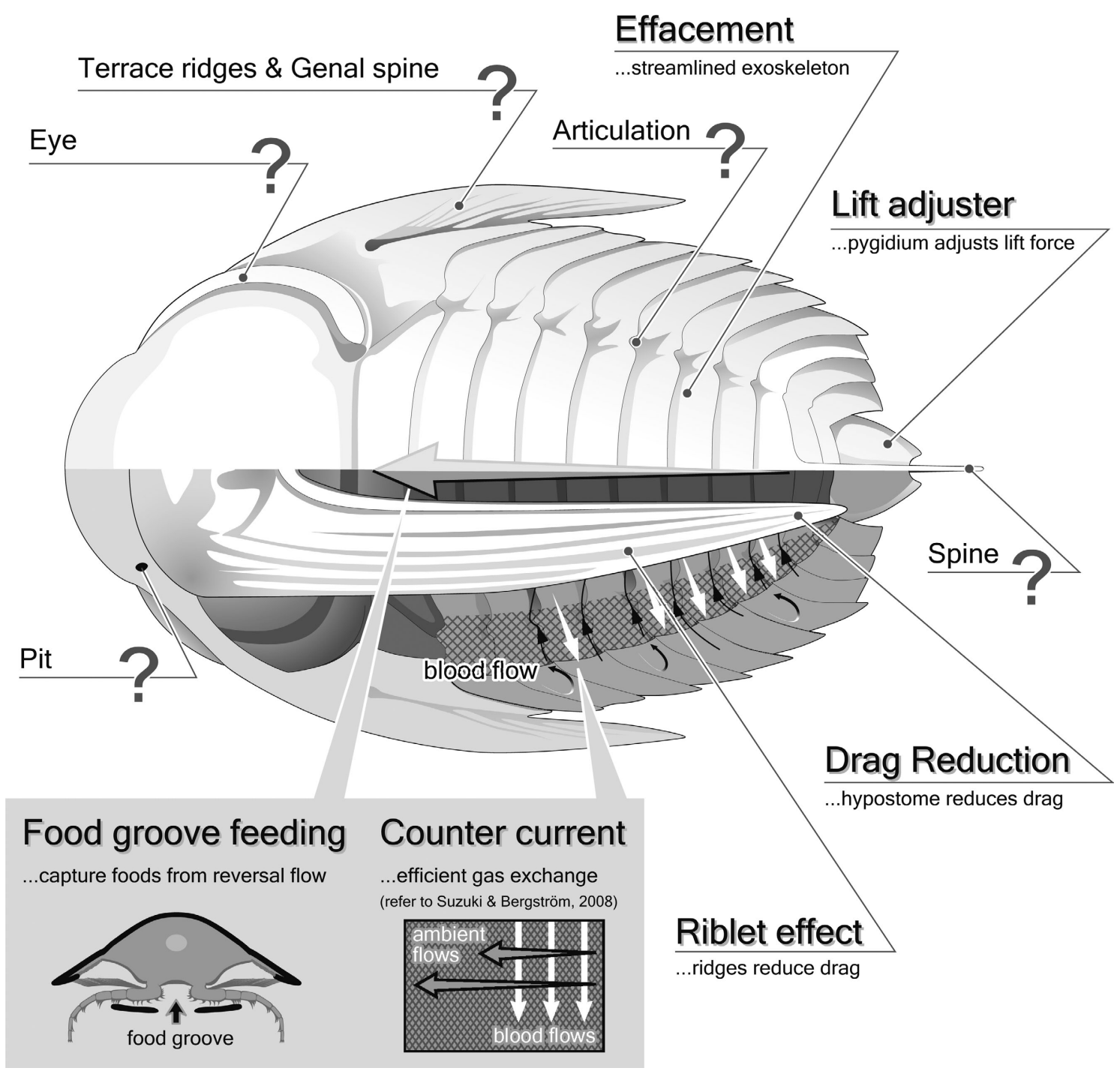

Figure 5. Morpho-functional diagram of Hypodicranotus. The exoskeletal characteristics enhance the swimming ability of the animal, thus generating vortex flows within the area surrounded by the exoskeleton (see also Shiino et al. 2012). This adaptation converts the apparently disadvantageous formation of vortex flows into a benefit for feeding and respiration, thus constituting a biological system with hybrid functionality. Some functional properties, indicated by question marks, remain uncertain.

an unknown role other than trilobite volvation. Given the morphological disparity of terrace ridges on the hypostome between Remopleurides and Hypodicranotus, there is a major difference in adaptive features between the two. The unusual swimming trilobite, Hypodicranotus exemplifies morphological adaptation for efficient swimming performance while maintaining homeostasis.

Considering that animals integrate a variety of biological performance traits within their bodies, other morphological features with functional roles can be understood in terms of their relevant autecological properties (Fig. 5). During swimming, Hypodicranotus may depend on its eyes to determine its swimming direction and speed in response to ambient conditions. Therefore, the visual system should be adapted to faster swimming near the sea bottom because arthropods optimise their sensory traits in response to relevant adaptive strategies (e.g., Clarkson 1967, 1975; McCormick \& Fortey 1999; Schoenemann et al. 2010; Ono 2011). The strip-like visual surface of the eyes with many thousands of tiny lenses arranged in an almost vertical fashion (Whittington 1952) enables it to have lateral and forward view like other benthic trilobites and to see its surroundings in high resolution. This may partially support our hypothesis. Additional morphological characteristics, such as the pattern of terrace ridges, the form of the genal spines, the mode of articulation and the prominent thoracic mid-axial spine located on the eighth thoracic segment, may also have contributed to the swimming performance of the animal as well as to its homeostasis. A pair of pits 
on the ventral side of the cephalic doublure may have played a role in an unknown biological system, possibly related to a specific behaviour. Future biomechanical analyses will help to elucidate how the hybrid functionality of the remopleuridid morphology has evolved under genetic and autecological constraints.

\section{Acknowledgements}

We are grateful to Jessica Cundiff and Tsuyoshi Takahashi (Harvard University) for a greatly appreciated arrangement to make plaster figures of fossil specimens. We thank Brigitte Schoenemann (The University of Bonn) and Rudy Lerosey-Aubril (Senckenberg Research Institute) for thorough discussions, critical comments and encouragement at TRILO 2012. We thank Paul Hille (Utrecht University) for providing information of fossil specimens. Thanks are also due to two reviewers, Richard Fortey (Natural History Museum) and Nigel Hughes (University of California Riverside). Funding was provided in part by the Kurata Grants from the Kurata Memorial Hitachi Science and Technology Foundation, and by the JSPS KAKENHI Grant Number 24840018 and 25630047.

\section{References}

Abe, K., Kondo, T. \& Nagano, Y. 1995. A new turbulence model for predicting fluid flow and heat transfer in separating and reattaching flows-II. Thermal field calculations. International Journal of Heat and Mass Transfer 38(8), 1467-1481. DOI 10.1016/0017-9310(94)00252-Q

ABE, K., Nagano, Y. \& Kondo, T. 1992. An improved $k-\varepsilon$ model for a prediction of turbulent flows with separation and reattachment. Transactions of Japan Society of Mechanical Engineers Series B, 58(554), 3003-3010. [in Japanese with English abstract] DOI 10.1299/kikaib.58.3003

AdKINS, D. \& YAN, Y.Y. 2006. CFD Simulation of fish-like body moving in viscous liquid. Journal of Bionic Engineering 3(3), 147-153. DOI 10.1016/S1672-6529(06)60018-8

Amati, L., Armitage, J.L., Middlebrook, N.R. \& Smith, A.K. 2009. Geographic and environmental distribution of two late Ordovician Remopleuridid trilobites and their implications for lifestyle. Geological Society of America Abstracts with Programs 41, 89.

Amati, L. \& Westrop, S.R. 2006. Sedimentary facies and trilobite biofacies along an Ordovician shelf to basin gradient, Viola Group, south-central Oklahoma. Palaios 21(6), 516-529. DOI 10.2110/palo.2006.p06-069

Azuma, A. 2006. The Biokinetics of Flying and Swimming. 518 pp. American Institute of Aeronautics and Astronautics, Inc., Reston.

Bergström, J. 1969. Remarks of the appendages of trilobites. Lethaia 2(4), 395-414.

DOI 10.1111/j.1502-3931.1969.tb01259.x

Bergström, J. 1973. Organization, life, and systematics of trilobites. Fossils and Strata 2, 1-69.
Bertram, V. 2000. Practical Ship Hydrodynamics. 270 pp. Butterworth-Heinemann, Oxford.

Boucot, A.J. 1981. Principles of Benthic Marine Paleoecology. 463 pp. Academic Press, New York.

Brett, C.E., Whiteley, T.E., Allison, P.A. \& Yochelson, E.L. 1999. Walcott-Rust Quarry: Middle Ordovician trilobite Konservat-Lagerstätten. Journal of Paleontology 73(2), 288-305.

Briggs, D.E.G., Dalingwater, J.E. \& Selden, P.A. 1991. Biomechanics of locomotion in fossil arthropods, 37-56. In RAYNER, J.M.V. \& WootTon, R.J. (eds) Biomechanics in Evolution. Cambridge University Press, Cambridge.

CHAN, T.Y. 1998. Shrimps and prawns, 851-971. In CARPENTER, K.E. \& NIEM, V.H. (eds) FAO species identification guide for fishery purposes. The living marine resources of the Western Central Pacific, volume 2. Cephalopods, Crustaceans, Holothurians and Sharks. FAO, Rome.

Chatterton, B.D.E. \& Speyer, S.E. 1997. Ontogeny, 173-247. In KAESLER, R.L. (ed.) Treatise on invertebrate paleontology. Part O, Arthropoda 1, Trilobita, Revised. The Geological Society of America \& The University of Kansas, Boulder \& Lawrence.

Clarkson, E.N.K. 1967. Environmental significance of eye-reduction in trilobites and recent arthropods. Marine Geology 5(5-6), 367-375. DOI 10.1016/0025-3227(67)90046-1

Clarkson, E.N.K. 1975. The evolution of the eye in trilobites. Fossils and Strata 4, 7-31.

Clarkson, E.N.K. \& Ahlberg, P. 2003. Ontogeny and structure of a new, miniaturised and spiny olenid trilobite from southern Sweden. Palaeontology 45(1), 1-22.

DOI 10.1111/1475-4983.00224

Dineshbabu, A.P. \& Manissery, J.K. 2009. Food and feeding of the ridgeback shrimp, Solenocera choprai Nataraj, along Karnataka coast. Indian Journal of Fisheries 56(1), 21-26.

Dorit, R.L., WALKer, W.F. \& BARNes, R.D. 1991. Zoology. 1009 pp. Saunders College Publishing, Philadelphia.

Esteve, J., Zamora, S., Gozalo, R. \& Liñán, E. 2010. Sphaeroidal enrolment in middle Cambrian solenopleuropsine trilobites. Lethaia 43(4), 478-493. DOI 10.1111/j.1502-3931.2009.00205.x

Esteve, J., Hughes, N.C. \& Zamora, S. 2011. Purujosa trilobite assemblage and the evolution of trilobite enrollment. Geology 39(6), 575-578. DOI 10.1130/G31985.1

Feist, R., Lerosey-Aubril, R. \& Johnson, R. 2010. Coaptative devices, enrollment, and life habits in Paralejurus, a particular case in scutelluid trilobites. Palaeobiodiversity and Palaeoenvironments 90(2), 125-137.

DOI 10.1007/s12549-010-0025-1

ForTEY, R.A. 1985. Pelagic trilobites as an example of deducing the life habits of extinct arthropods. Transactions of the Royal Society of Edinburgh, Earth Sciences 76, 219-230. DOI $10.1017 / \mathrm{S} 0263593300010452$

ForTEY, R.A. 2004. The lifestyles of the trilobites. American Scientist 92(5), 446-453. DOI 10.1511/2004.49.944

Fortey, R.A. \& Chatterton, B.D.E. 1988. Classification of the trilobite Suborder Asaphina. Palaeontology 31(1), 17-19.

Fortey, R.A. \& Owens, R.M. 1990. Trilobites, 121-142. In McNamara, K.J. (ed.) Evolutionary Trends. The University of Arizona Press, Tuscon. 
FRYER, G. 1988. Studies on the functional anatomy and biology of the Notostraca (Crustacea: Branchiopoda). Philosophical Transactions of the Royal Society of London Series B, Biological Sciences 321, 27-124. DOI 10.1098/rstb.1988.0091

FujIWARA, S. \& HutChinson, J.R. 2012. Elbow joint adductor moment arm as an indicator of forelimb posture in extinct quadrupedal tetrapods. Proceedings of the Royal Society Series B, Biological Sciences 279(1738), 2561-2570. DOI 10.1098/rspb.2012.0190

Hansen, T., Nielsen, A.T. \& Bruton, D.L. 2011. Palaeoecology in a mud-dominated epicontinental sea: A case study of the Ordovician Elnes Formation, southern Norway. Palaeogeography, Palaeoclimatology, Palaeoecology 299(1-2), 348-362. DOI 10.1016/j.palaeo.2010.11.014

Hegna, T. 2010. The function of forks: Isotelus-type hypostomes and trilobite feeding. Lethaia 43(3), 411-419.

Holthuis, L.B. 1980. FAO species catalogue. Vol. 1. Shrimps and prawns of the world. An annotated catalogue of species of interest to fisheries. FAO Fisheries Synopsis 125(1), 1-271.

Hughes, N.C. 2007. The evolution of trilobite body patterning. Annual Review of Earth and Planetary Sciences 35, 401-434. DOI 10.1146/annurev.earth.35.031306.140258

Hughes, N.C. \& CHAPMAN, R.E. 1995. Growth and variation in the Silurian proetide trilobite Aulacopleura konincki and its implications for trilobite palaeobiology. Lethaia 28(4), 333-353. DOI 10.1111/j.1502-3931.1995.tb01824.x

JACKLYN, P.M. \& RITZ, D.A. 1986. Hydrodynamics of swimming in scyllarid lobsters. Journal of Experimental Marine Biology and Ecology 101(1-2), 85-99. DOI 10.1016/0022-0981(86)90043-2

JohANSEN, P.O. \& BATTEGARD, T. 1998. Observation on behavior and distribution of Natatolana borealis (Lilljeborg) (Crustacea, Isopoda). Sarsia 83(4), 347-360.

Kaji, T., Møller, O.S. \& Tsukagoshi, A. 2011. A bridge between original and novel states: ontogeny and function of "suction discs" in the Branchiura (Crustacea). Evolution \& Development 13(2), 119-126. DOI 10.1111/j.1525-142X.2011.00462.x

KunJu, M.M. 1970. Synopsis of biological data on the penaeid prawn Solenocera indica Nataraj, 1945. FAO Fisheries Report 4(57), 1317-1333.

LAND, M.F. 2000. On the functions of double eyes in midwater animals. Philosophical Transactions of the Royal Society Series B, Biological Sciences 355(1401), 1147-1150. DOI 10.1098/rstb.2000.0656

Lerosey-Aubril, R., Hegna, T.A. \& Olive, S. 2011. Inferring internal anatomy from the trilobite exoskeleton: the relationship between frontal auxiliary impressions and the digestive system. Lethaia 44(2), 166-184. DOI 10.1111/j.1502-3931.2010.00233.x

Lowry, J.K. \& DemPSEY, K. 2006. The giant deep-sea scavenger genus Bathynomus (Crustacea, Isopoda, Cirolanidae) in the Indo-West Pacific, 163-192. In Richer DE Forges, B. \& JusTine, J.-L. (eds) Tropical Deep-Sea Benthos, volume 24. Mémoires du Muséum national d'histoire naturelle 193.

Ludvigsen, R. \& Chatterton, B.D.E. 1991. The peculiar Ordovician trilobite Hypodicranotus from the Whittaker Formation, District of Mackenzie. Canadian Journal of Earth Sciences 28(4), 616-622. DOI 10.1139/e91-053
Marshall, N.J. \& Diebel, C. 1995. 'Deep-sea spiders' that walk through the water. The Journal of Experimental Biology 198(6), 1371-1379.

Matsui, T., Moriyama, T. \& Kato, R. 2011. Burrow plasticity in the deep-sea isopod Bathynomus doederleini (Crustacea: Isopoda: Cirolanidae). Zoological Science 28(12), 863-868. DOI 10.2108/zsj.28.863

Matsumura, Y. \& Yoshizawa, K. 2012. Homology of the internal sac components in the leaf beetle subfamily Criocerinae and evolutionary novelties related to the extremely elongated flagellum. Journal of Morphology 273(5), 507-518.

DOI 10.1002/jmor.11037

McCormick, T. \& Fortey, R.A. 1999. The most widely distributed trilobite species Ordovician Carolinites genacinaca. Journal of Paleontology 73(2), 202-218.

Nishino, H. \& SAKAI, M. 1996. Behaviorally significant immobile state of so-called thanatosis in the cricket Gryllus bimaculatus DeGeer: its characterization, sensory mechanism and function. Journal of Comparative Physiology A 179(5), 613-624. DOI 10.1007/BF00216126

Nikolaisen, F. 1988. The Middle Ordovician of the Oslo Region, Norway, 32. Trilobites of the family Remopleurididae. Norsk Geologisk Tidsskrift 62, 231-329.

Nowell, A.R.M., Jumars, P.A. \& ECKMAN, J.E. 1981. Effects of biological activity on the entrainment of marine sediments. Marine Geology 42, 133-153.

DOI 10.1016/0025-3227(81)90161-4

ONO, S. 2011. Adaptive design of trilobite compound eyes: Integration between their optical features and mode of growth. Fossils 89, 1-2. [in Japanese]

PARK, T.-Y. \& ChOI, D.K. 2011. Ontogeny of the Furongian (late Cambrian) remopleuridioid trilobite Haniwa quadrata Kobayashi, 1933 from Korea: implications for trilobite taxonomy. Geological Magagine 148(2), 288-303. DOI 10.1017/S0016756810000701

RASMussen, C.M.Ø. \& HARPER, D.A.T. 2011. Did the amalgamation of continents drive the end Ordovician mass extinctions? Palaeogeography, Palaeoclimatology, Palaeoecology 311(1-2), 48-62. DOI 10.1016/j.palaeo.2011.07.029

RupPERT, E.E., Fox, R.S. \& BARNES, R.D. 2003. Invertebrate Zoology: A Functional Evolutionary Approach, Seventh Edition. 963 pp. Brooks/Cole, Belmont.

Schoenemann, B., Clarkson, E.N.K., Ahlberg, P. \& Álvarez, M.E.D. 2008. A Furongian polymerid planktonic trilobite, 361-364. In Rábano, I., Gozalo, R. \& García-Bellido, D. (eds) Advances in trilobite research. Instituto Geológico y Minero de España, Madrid.

Schoenemann, B., Clarkson, E.N.K., Ahlberg, P. \& Álvarez, M.E.D. 2010. A tiny eye indicating a planktonic trilobite. Palaeontology 53(4), 695-701. DOI 10.1111/j.1475-4983.2010.00966.x

Schotte, M., Kensley, B.F. \& Shilling, S. 1995. World list of Marine, Freshwater and Terrestrial Crustacea Isopoda. National Museum of Natural History Smithsonian Institution, Washington D.C., USA. http://invertebrates.si.edu/isopod/

Shino, Y. 2010. Passive feeding in spiriferide brachiopods: an experimental approach using models of Devonian Paraspirifer and Cyrtospirifer. Lethaia 43(2), 223-231. DOI 10.1111/j.1502-3931.2009.00185.x 
Shino, Y. \& Kuwazuru, O. 2010. Functional adaptation of spiriferide brachiopod morphology. Journal of Evolutionary Biology 23(7), 1547-1557.

DOI 10.1111/j.1420-9101.2010.02024.x

Shino, Y. \& Kuwazuru, O. 2011a. Theoretical approach to the functional optimisation of spiriferide brachiopod shell: Optimum morphology of sulcus. Journal of Theoretical Biology 276(1), 192-198. DOI 10.1016/j.jtbi.2011.02.004

Shino, Y. \& Kuwazuru, O. 2011b. Comparative experimental and simulation study on passive feeding flow generation in Cyrtospirifer. Memoirs of the Association of Australasian Palaeontologists 41, 1-8.

Shino, Y., Kuwazuru, O., Suzuki, Y. \& ONO, S. 2012. Swimming capability of the remopleuridid trilobite Hypodicranotus striatus: Hydrodynamic functions of the exoskeleton and the long, forked hypostome. Journal of Theoretical Biology 300(1), 29-38. DOI 10.1016/j.jtbi.2012.01.012

Shino, Y., Kuwazuru, O. \& Yoshikawa, N. 2009. Computational fluid dynamics simulations on a Devonian spiriferid Paraspirifer bownockeri (Brachiopoda): Generating mechanism of passive feeding flows. Journal of Theoretical Biology 259(1), 132-141. DOI 10.1016/j.jtbi.2009.02.018

SHIINO, Y. \& SUZUKI, Y. 2007. Articulatory and musculatory systems in a Permian concavo-convex brachiopod Waagenoconcha imperfecta Prendergast, 1935 (Productida, Brachiopoda). Paleontological Research 11(3), 265-275. DOI 10.2517/1342-8144(2007)11[265:AAMSIA]2.0.CO;2

ShinN, Y. \& Suzuki, Y. 2011. The ideal hydrodynamic form of the concavo-convex productide brachiopod shell. Lethaia 44(3), 329-343. DOI 10.1111/j.1502-3931.2010.00243.x

Shinno, Y., Suzuki, Y. \& Kobayashi, F. 2011. Sedimentary history with biotic reaction in the Middle Permian shelly se- quence of the Southern Kitakami Massif, Japan. Island Arc 20(2), 203-220. DOI 10.1111/j.1440-1738.2011.00760.x

SNELGRove, P.V.R. 1998. The biodiversity of macrofaunal organisms in marine sediments. Biodiversity and Conservation 7(9), 1123-1132. DOI 10.1023/A:1008867313340

SUZUKI, Y. \& BERGSTRÖM, J. 2008. Respiration in trilobites: a reevaluation. GFF 130(4), 211-229. DOI 10.1080/11035890809452774

SuZuki, Y., Kondo, A. \& Bergström, J. 2008. Morphological requirements in limulid and decapod gills: A case study in deducing the function of lamellipedian exopod lamellae. Acta Palaeontologica Polonica 53(2), 275-283.

DOI 10.4202/app.2008.0208

Vogel, S. 1994. Life in Moving Fluids. 467 pp. Princeton University Press, Princeton.

Walcott, C.D. 1875. New species of trilobite from the Trenton Limestone at Trenton Falls, New York. Cincinnati Quarterly Journal of Science 2, 347-349.

Whittington, H.B. 1952. A unique remopleuridid trilobite. Breviora 4, 1-9.

Whittington, H.B. 1959. Silicified Middle Ordovician trilobites: Remopleurididae, Trinucleidae, Raphiophoridae, Endymioniidae. Bulletin of The Museum of Comparative Zoology 121(8), 369-496.

Whittington, H.B. 1975. Trilobites with appendages from the Middle Cambrian, Burgess Shale, British Columbia. Fossils and Strata 4, 97-136.

Zhang, X. \& BARnes, C.R. 2007. Late Ordovician to Early Silurian conodont faunas from the Kolyma Terrane, Omulev Mountains, northeast Russia, and their paleobiogeographic affinity. Journal of Paleontology 81(3), 490-512.

DOI 10.1666/05077.1 